\title{
CNS side effects of immune checkpoint inhibitors: preclinical models, genetics and multimodality therapy
}

\author{
Gwendolyn J McGinnis ${ }^{1,2}$ \& Jacob Raber*,1,2,3,4 \\ ${ }^{1}$ Department of Radiation Medicine, Oregon Health \& Science University, OR, USA \\ ${ }^{2}$ Department of Behavioral Neuroscience, Oregon Health \& Science University, OR, USA \\ ${ }^{3}$ Department of Neurology, Oregon Health \& Science University, OR, USA \\ ${ }^{4}$ Division of Neuroscience, Oregon National Primate Research Center, 505 NW 185th Ave, Beaverton, OR 97006, USA \\ * Author for correspondence: Tel.: +1 503494 1524; Fax: +1 503494 6877; raberj@ohsu.edu
}

Following cancer treatment, patients often report behavioral and cognitive changes. Novel cancer immunotherapeutics have the potential to produce sustained cancer survivorship, meaning patients will live longer with the side effects of treatment. Given the role of inflammatory pathways in mediating behavioral and cognitive impairments seen in cancer, we aim in this review to discuss emerging evidence for the contribution of immune checkpoint blockade to exacerbate these CNS effects. We discuss ongoing studies regarding the ability of immune checkpoint inhibitors to reach the brain and how treatment responses to checkpoint inhibitors may be modulated by genetic factors. We further consider the use of preclinical tumor-models to study the role of tumor status in CNS effects of immune checkpoint inhibitors and multimodality therapy.

First draft submitted: 8 May 2017; Accepted for publication: 9 August 2017; Published online: 15 September 2017

Keywords: checkpoint inhibitor immunotherapy • neuroinflammation • radiotherapy

Patients undergoing cancer treatment and cancer survivors commonly describe behavioral alterations and cognitive impairments [1-3]. Symptoms can include behavioral changes, such as fatigue, depression and increased anxiety, as well as cognitive impairments, such as difficulty concentrating and memory impairments [4]. These impairments can have a major impact on quality of life and up to 35\% of patients report symptoms lasting months or years after finishing cancer treatment [5], making it one of the most frequently reported symptoms in cancer care. As novel cancer therapeutics improve overall survival, more and more patients are living longer with the side effects of cancer treatment, increasing the importance of understanding and predicting long-term quality of life outcomes. These effects are often collectively identified as 'chemobrain', given their historical association with cytotoxic chemotherapy. However, the mechanisms underlying cancer-related cognitive and behavioral problems are likely multifactorial.

Much of the recent literature on cancer-related cognitive and behavioral impairments has focused on the contribution of immune activation in the CNS [6-9]. Although the CNS has historically been considered an immuneprivileged site, it is increasingly evident that systemic immune activation can mediate central neuroinflammation and has downstream behavioral and cognitive effects [10]. Neuroinflammatory responses mediate symptoms and progression in a number of neurological conditions. There is evidence of enhanced proinflammatory profile linked with fatigue, major depression, memory complaints, behavioral deficits, pathogenesis of cerebral ischemia and Alzheimer's disease [11]. In Alzheimer's disease, innate immune activation and microglia-mediated neuroinflammatory responses promote initiation and progression of disease [12]. These differences can be seen systemically through increased expression of the key proinflammatory mediators TNF- $\alpha$, IL- $1 \beta$, IFN- $\gamma$ and IL- 6 , but also in an altered immune environment in the CNS. For example, the brains of depressed patients completing suicide show increased microglial activation and macrophage recruitment [13]. Causative role of these inflammatory cytokines and chemokines is supported by data that show administration can induce depressive-like behavior [8]. Immune 
Table 1. Adverse events of checkpoint inhibitor immunotherapy with potential to alter cognition and behavioral performance.

\begin{tabular}{|c|c|c|c|c|c|c|c|c|c|c|c|}
\hline \multirow[t]{2}{*}{ Study (year) } & \multirow[t]{2}{*}{ Treatment } & \multirow[t]{2}{*}{$\mathbf{n}$} & \multicolumn{2}{|c|}{ Fatigue (\%) } & \multicolumn{2}{|c|}{ Decreased appetite (\%) } & \multicolumn{2}{|c|}{ Pyrexia (\%) } & \multicolumn{2}{|c|}{$\begin{array}{l}\text { Endocrine abnormality } \\
\qquad(\%)^{\dagger}\end{array}$} & \multirow[t]{2}{*}{ Ref. } \\
\hline & & & Total & Grade 3-4 & Total & Grade 3-4 & Total & Grade 3-4 & Total & Grade 3-4 & \\
\hline $\begin{array}{l}\text { Hodi et al. } \\
\text { (2010) }\end{array}$ & Ipilimumab & 131 & 42 & 6.9 & 26.7 & 1.5 & 12.2 & 0 & 7.6 & 2.3 & [99] \\
\hline Garon et al. & Pembrolizumab & 495 & 19.4 & 0.8 & 10.5 & 1.0 & 4.20 & 0.60 & 6.90 & 0.20 & [100] \\
\hline $\begin{array}{l}\text { Weber et al. } \\
\text { (2015) }\end{array}$ & Nivolumab & 268 & 24 & 1 & 5 & 0 & - & - & - & - & [101] \\
\hline
\end{tabular}

†Includes hypothyroidism, hypopituitarism, hypophysitis, and adrenal insufficiency.

activation has also been investigated as part of the pathogenesis of cancer- and cancer treatment-related cognitive and behavioral impairments. The inflammatory challenge of a tumor itself can contribute to the behavioral alterations and cognitive impairments seen with cancer and cancer treatment [14-17]. The role of neuroinflammation in cancer-related cognitive impairment has become especially important with development of novel treatments combining radiation treatment and immunotherapy. These treatments demonstrate remarkable efficacy with respect to tumor outcomes by enhancing the proinflammatory environment in the tumor, but how they may influence the immune environment in the brain, and thus behavioral and cognitive performance, is less clear. As yet, very little is understood about the effects of these treatments on the brain, either in healthy individuals or in individuals with tumors.

Typical symptoms seen with immunotherapy, which overlap with those of sickness behavior include fatigue, anorexia and pain (Table 1). Other adverse events following immunotherapy treatment which may contribute to symptoms of sickness-like behavior include endocrine abnormalities, such as hypothyroidism, hypopituitarism, hypophysitis and adrenal insufficiency (Table 1). From an evolutionary perspective, sickness behavior is an adaptive response to conserve energy to promote healing [18,19]. Conserving energy might involve anhedonia, increased pain sensitivity, social avoidance and reduced exploratory drive, basically symptoms of depressive behavior and increased anxiety levels [20].

Of particular interest in considering checkpoint inhibitor immunotherapy is the significant role of the underlying genetic substrate. Although these novel therapeutics have the promising ability to achieve sustained cure, they are only able to do so in a small subset of patients. Understanding biomarkers and improving our ability to predict tumor-related outcomes will be important to efficiently utilize these therapeutics. However, the same will likely prove true in the consideration of adverse events. Certain individuals are likely more susceptible to the behavioral and cognitive impairments imparted by enhanced immune activation following immune checkpoint blockade. By looking at known genetic risk factors, we can begin to understand individual susceptibility to CNS side effects and improve our ability to deliver personalized medicine.

Animal tumor models present the opportunity to study the consequences of cancer and cancer treatment on brain function. Behavioral performance and cognition in response to therapeutic intervention have rarely been studied in mouse models of cancer, which provide the important opportunity to investigate changes at the behavioral, physiological and molecular level. However, these models provide insight into how the systemic and central environments respond differently in the presence versus the absence of a tumor. These differential effects have important implications for the approach to prolonged treatment with checkpoint inhibitor immunotherapy, especially with regard to managing CNS side effects.

Here, we review early evidence suggesting the role of checkpoint inhibitor immunotherapy, especially when combined or as part of multimodality therapy, to exacerbate the behavioral alterations and cognitive impairments seen with cancer and cancer treatment. We consider early evidence for central activity of peripherally administered immunotherapy and how immune activation can be increased by pairing checkpoint inhibitors or adding complementary therapies like peripheral radiation or tumor vaccine. Given the variable tumor response to checkpoint inhibitor immunotherapy, we further consider how cognitive and behavioral side effects may also be considered in the framework of responders versus nonresponders. We discuss the potential role of the underlying genetic substrates and in particular the known genetic risk factor, apolipoprotein $\mathrm{E}$ (apoE), in mediating these effects. Finally we address the critical role of preclinical models in exploring these complicated systemic effects and how 
specifically tumor-bearing models may be used to investigate the cognitive and behavioral side effects of cancer treatment.

\section{Immune checkpoint inhibitors \& the CNS}

Immune checkpoint inhibitors represent one of the most promising recent innovations in cancer therapeutics and hold the promise to significantly increase rates of cancer survivorship. These treatments have been developed based on findings that show that the immune environment within a tumor is associated with clinical outcome in a variety of cancers. A greater proinflammatory polarization of a tumor microenvironment is associated with increased survival, while anti-inflammatory tumor profiles are associated with limited survival [21,22]. Checkpoint inhibitor immunotherapies aim to induce a proinflammatory environment characterized by increased immune infiltrates into tumors. The most studied immune checkpoint blockade therapies include monoclonal antibodies targeted against CTLA-4, PD-1 and PD-L1. CTLA-4 is a member of the immunoglobulin superfamily that is expressed on the surface of helper $\mathrm{T}$ cells and other $\mathrm{T}$ cells such as regulatory $\mathrm{T}$ cells and suppresses immune activation by transmitting an inhibitory signal to T cells. PD-1 is a cell-surface receptor that belongs to the immunoglobulin superfamily and is expressed on T cells and pro-B cells. Anti-CTLA- 4 has been shown to induce a proinflammatory environment characterized by increased tumor immune infiltrates [23]. Recent evidence suggests that PD-1 influences T-cell activation through regulation of the T-cell receptor costimulatory molecule CD28 [24,25]. There is mounting clinical evidence for the utility of these therapeutics in an expanding spectrum of tumors, including melanoma, non-small-cell lung cancer, renal cell carcinoma, ovarian cancer, bladder cancer, head and neck cancer and gastric cancer [26]. These treatments demonstrate remarkable efficacy with respect to tumor outcomes, but how they may influence the immune environment in the brain, and thus behavior, is unknown.

Preclinical animal studies suggest that checkpoint inhibitors might be able to pass the blood-brain barrier or at least result in a therapeutic response in the brain. For example, anti-CTLA- 4 antibodies were beneficial in mice with SMA-650 intracranial tumors [27]. In addition, when combined with radiation treatment, anti-PD-1 antibodies improved survival in mice with GL261 intracranial tumors [28]. Consistent with these results, combined treatment with anti-CTLA-4 and anti-PD1 antibodies improved survival in glioblastoma mouse models [29]. However, the beneficial effects of checkpoint inhibitors in the context of brain tumors are not necessarily due to the ability of checkpoint inhibitors to pass the blood-brain barrier as peripheral immune activation might be sufficient to trigger a central immune response.

There is limited research describing the effects of immune checkpoint inhibitors on the brain. Recent trials investigating the effects of checkpoint inhibitors on brain metastases can shed some light on the ability of these peripherally delivered therapeutics to influence the immune system in the CNS. Several trials in non-small-cell lung cancer demonstrate partial or complete response of brain metastases to anti-PD-1 checkpoint blockade at rates similar to those expected for nonbrain diseases sites [30-32]. In another example of the central action of immune checkpoint blockade, Baruch $e$ t al. describe increased cerebral amyloid plaque clearance following PD-1 immune checkpoint blockade in a mouse model of Alzheimer's disease [33]. In this model, the effect in the brain was interferon-dependent. However, long-term, continuous treatment is required to see effects in both tumor outcomes and cerebral amyloid- $\beta$ plaque clearance, suggesting that immune checkpoint blockade must be chronic to exert central effects.

Early clinical data describe behavioral and cognitive outcomes following immune checkpoint blockade in the setting of cancer treatment. Severe immune-related side effects of therapy, which are expected to negatively affect cognition include endocrine effects, fatigue, headache, fever and loss of appetite [34]. Episodes of transient but severe cognitive dysfunction, including memory loss, have been described [30,35]. However, there remains a gap in how these therapies modulate cognitive impairments and behavioral alterations.

While both checkpoint inhibitors and proinflammatory cytokines activate the immune system, the effects of checkpoint inhibitors on the immune system are distinct. For example, CTLA- 4 antibodies decrease the number of regulatory $\mathrm{T}$ cells, which is mediated by monocytes [36], while PD-1 antibodies increase the activity of T cells [37].

\section{The role of genetic factors in modulating the treatment response \& CNS side effects}

Despite the early promise of immune checkpoint blockade, it is becoming clear that only a small subset of patients respond to single agent therapy $[38,39]$. Therefore, the use and refinement of biomarkers predicting treatment responses to checkpoint inhibitor therapies is critical. In general, patients with tumors that produce high levels of PD-L1 respond well to the anti-PD1/anti-PD-L1 checkpoint inhibitor treatment while those with tumors 


\begin{tabular}{|c|c|c|c|c|c|}
\hline Study (year) & Sample & Study design & Treatment modalities & ApoE4 association & Ref \\
\hline \multicolumn{6}{|c|}{ Active disease, undergoing treatment } \\
\hline Vardy et al. (2015) & Colorectal cancer & Longitudinal & Chemotherapy & No association & {$[102$} \\
\hline Amidi et al. (2016) & Testicular cancer & Longitudinal & Surgery and/or chemotherapy & $\begin{array}{l}\text { ?Cognitive impairment after } \\
\text { chemotherapy }\end{array}$ & {$[103$} \\
\hline Koleck et al. (2014) & Breast cancer & Longitudinal & $\begin{array}{l}\text { Hormonal therapy and/or } \\
\text { chemotherapy }\end{array}$ & $\begin{array}{l}\text { ?Cognitive impairment before } \\
\text { and after treatment (verbal } \\
\text { learning and memory, executive } \\
\text { function) }\end{array}$ & {$[104$} \\
\hline Ahles et al. (2014) & Breast cancer & Longitudinal & Chemotherapy & $\begin{array}{l}\text { ?Cognitive impairment } \\
\text { (processing speed, working } \\
\text { memory) }\end{array}$ & {$[105$} \\
\hline \multicolumn{6}{|l|}{ Post-treatment } \\
\hline Lengacher et al. (2015) & Breast cancer & Prospective RCT & $\begin{array}{l}\text { Radiation and/or } \\
\text { chemotherapy }\end{array}$ & ?Cognitive impairment & {$[106$} \\
\hline Ahles et al. (2003) & Breast cancer, lymphoma & Cohort & Chemotherapy & $\begin{array}{l}\text { ?Cognitive impairment (visual } \\
\text { memory, spatial ability) }\end{array}$ & {$[107$} \\
\hline
\end{tabular}

that produce low levels of PD-L1 do not, but additional biomarkers to predict treatment responses are needed [40]. While colorectal cancer patients with high microsatellite instability have a higher probability to respond to anti-PD1 antibody treatment, bladder cancer patients with a high tumor mutational burden (TMB) responded better to antiPD1 antibody therapy than those with a low TMB and in those patients TMB turned out to be a better predicting biomarker of treatment response than microsatellite instability [40]. Additional considerations are predicting and working around primary and acquired drug resistance to immunotherapy, potentially by using additional treatments focused around neoantigens [41], defining the optimal daily timing of treatment [42], and biocompatible polymers and other scaffolds for optimal delivery to enhance the effectiveness and half-life of the bioactive treatments [43]. Further, as learned from trials using chimeric antigen receptor T-cell therapy, a hyperactive immune system might be fatal in the case cerebral edema develops and therefore these potential toxicities need to be carefully worked around [44].

Genetic factors are likely involved in critically modulating not only the therapeutic response but also the side effects cancer patients experience who are treated with immune checkpoint blockade or other forms of immunotherapy. These genetic factors might be known modulators of the immune response in other clinical conditions. One gene that might play a role in this context is the one encoding apolipoprotein $\mathrm{E}$ (apoE). apoE plays an important role in the metabolism and redistribution of lipoproteins and cholesterol. Distinct alleles $(\varepsilon 2, \varepsilon 3$ and ६4) encode human apoE isoforms, apoE2, apoE3 and apoE4. These proteins are involved in cholesterol metabolism throughout the body and the brain. In the brain, apoE has been implicated in development, regeneration, neurite outgrowth and neuroprotection. Compared with apoE2 and apoE3, apoE4 increases the risk of learning and memory impairment following cardiac bypass surgery or traumatic brain injury, and of developing cardiovascular disease and Alzheimer's disease [45-49]. ApoE isoforms have differential effects on the immune system [50] and this in turn might mediate the CNS injury seen in immune-related clinical conditions [51]. For example, in HIV patients, those carrying apoE4 showed excess dementia and peripheral neuropathy [52] and enhanced HIV-1 cell entry associated with accelerated disease progression [53]. This might be a general theme, as enhanced viral replication and related injury in the brain has been seen in the context of HSV-1 in apoE4 carriers [54].

To determine whether there is an association between plasma cholesterol levels and cancer risk, Trompet $e t$ al. performed a Mendelian randomization study in 2913 participants during a 3-year follow-up period [55]. In this study, carriers with apoE2 did not have increased cancer incidence as compared with those with apoE4. In contrast, apoE 4 was identified as a risk factor for prostate cancer in a multicountry ecological study [56], while apoE2 was identified as a reducing gastric cancer [57]. In prostate cancer cell lines, $\varepsilon 3 / \varepsilon 3$ and $\varepsilon 3 / \varepsilon 4$ carrying cell lines were nonaggressive while cell lines carrying $\varepsilon 2 / \varepsilon 4$ were aggressive [58]. ApoE might also be a direct target for treating cancer; apoE was shown to be required for cell proliferation and survival in ovarian cancer [59]. In addition, multiple trials have identified apoE4 as a risk factor for cognitive impairment following cancer or cancer treatment (See Table 2). Clearly, more research is needed, particularly regarding the hypothesis whether or not cancer patients with 
Figure 1. Example of an experimental paradigm of a preclinical mouse study to assess cancer and cancer treatment-related behavioral alterations and cognitive impairments.

Mouse models of cancer and cancer treatment-related behavioral alterations and cognitive impairments allow for exploration of the role of genetic factors, specific tumors and various interventions, including immune checkpoint blockade and radiotherapy. Mouse models not only allow for controlled studies of each of the above factors but can be used to generate data on invasive outcomes under controlled environmental conditions unavailable in human studies in a clinical environment.

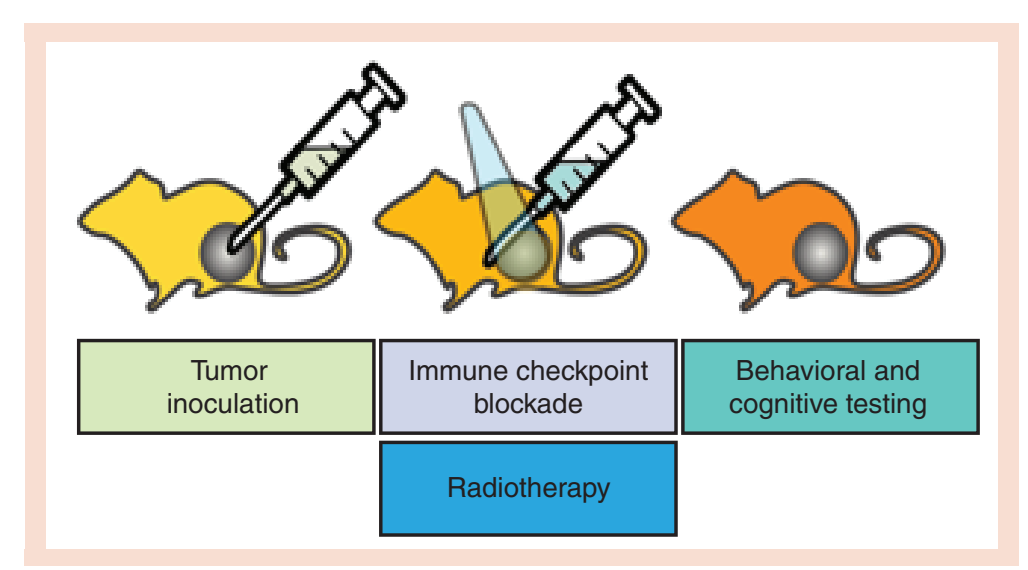

apoE4 have a reduced treatment response and more severe side effects than those without apoE4 [60].

Other genetic risk factors that would be expected to modulate the treatment response and CNS side effects of immunotherapy are polymorphisms related to depression and anxiety phenotypes, including those involving the serotonin transporter [61], IL-1 $\beta$, IL-6 [62] and nitric oxide synthase-1 [63]. In addition, genetic risk factors for CNS side effects on type I interferon treatment [64] would also be expected to modulate the treatment response and CNS side effects of immunotherapy.

\section{Preclinical models to study CNS effects of immune checkpoint inhibitors \& of combined immune checkpoint inhibitors with peripheral irradiation}

Historically, most preclinical studies to determine CNS effects of cancer treatments, including irradiation [65] and chemotherapy [66], have focused on healthy tissue injury as a result of the treatment in animals who do not bear tumors. While we learned from those studies, it becomes increasingly clear that especially in the context of immune activation treatments, it is important to also consider the effects of the tumor on the immune system. In the brain, immune checkpoint inhibitors might have differential effect in the presence and absence of tumors. Figure 1 illustrates an example of an experimental paradigm of such a preclinical model. We recognize that for practical purposes, it might not be feasible or reasonable to include all distinct experimental groups outlined. Depending on the question asked, this might also not be required. For example, it might be sufficient to consider comparing two genotypes, each involving tumor-bearing mice, with and without the combined treatments. So in this way, there would be only two experimental groups per genotype. This simplification of the experimental paradigm would not allow assessing how the phenotype of the tumor-bearing mice compares to that of mice lacking tumors or assessing the effects of the individual components of the cancer treatment but that could be assessed in follow-up studies. A simplification of the experimental paradigm as described above would also be practical based on the complexity of the immune system and multiple genetic models would be of interest in the context of immunotherapy. The complexity of the immune system and the beneficial versus detrimental role of immune activation in the brain in the context of neurodegenerative conditions is illustrated by the discussed role of the complement system and activated microglia [67] in pathology, metabolic function, autophagy and synaptic pruning [68].

Several studies have used tumor-bearing mouse models to assess effects of therapeutic interventions on the brain (See Table 3). Preclinical data support this notion and a particular role for altered levels of cytokines, chemokines and microglial activation in brain areas such as the hippocampus, which play an important role in cognition and are sensitive to cancer treatment effects [69], in this process [70] (See Table 3). The finding of enhanced microglial activation is important given previous findings that microglial activation, especially when chronic, is associated with neurodegenerative conditions [12]. The concept of neuroinflammation, illustrated in Figure 2, is not restricted to immunotherapy but also seen following irradiation only [71-75], and actually in various neurological and neurodegenerative conditions $[76,77]$. 


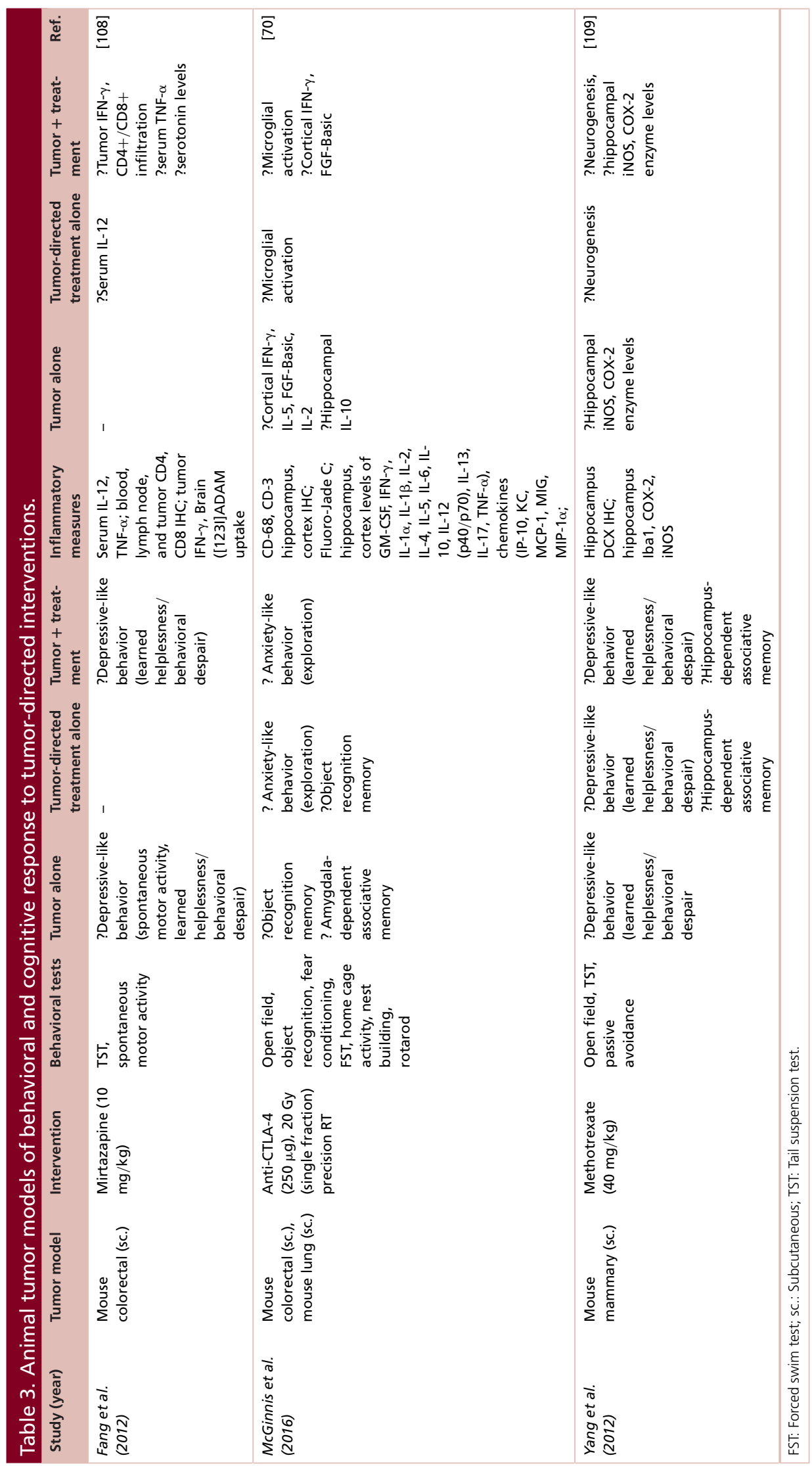




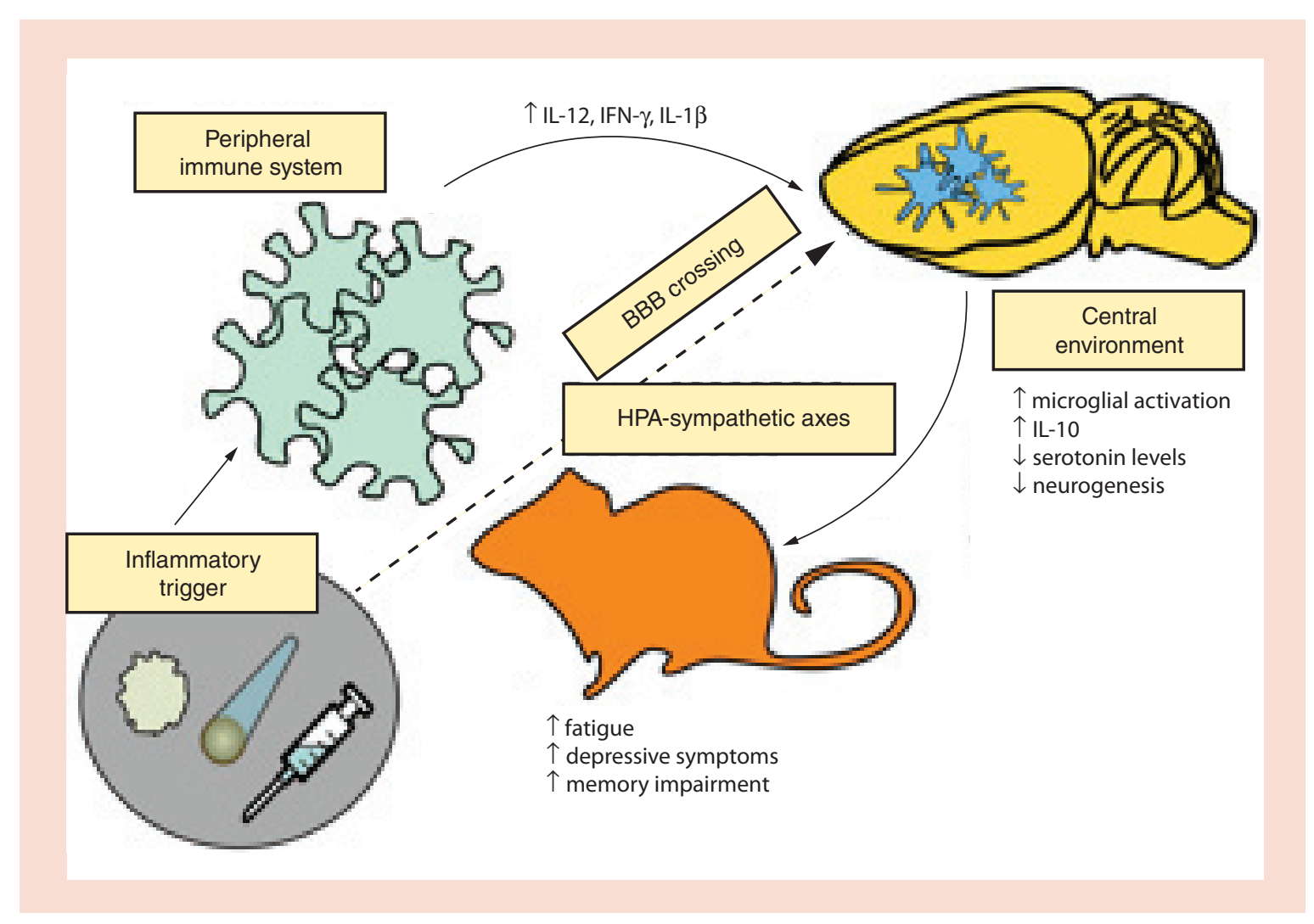

Figure 2. Activation of the peripheral and central immune system is beneficial in treating tumorigenesis.

However, this activation might cause cancer-related alterations in behavioral performance and cognitive impairments. There is increasing evidence supporting a role for peripheral and central inflammatory response to the tumor, radiation and immunotherapy in mediating CNS injury.

Whole brain irradiation has long been recognized as a mediator of behavioral alterations and cognitive impairments. Following cranial irradiation in rodent models, impairment in hippocampus-dependent cognition, such as contextual fear and spatial learning and memory, as well as neurogenesis in the dentate gyrus, have been reported [7880]. Immune activation may contribute to these effects [81,82]. In fact, the role of the immune system in the efficacy of radiation therapy is being increasingly targeted for therapeutic effect. The abscopal response, the response of a distant, out-of-field tumor to localized radiation therapy delivered elsewhere, provides additional support for the role of the local immune system in mediating a systemic immune response following radiation [83-87].

In preclinical models, neither anti-CTLA-4 nor anti-PD-1 are typically adequate as single agent antitumor therapies, but require vaccination or irradiation to initiate an immune response [88]. Therefore, combined treatment with multiple immune checkpoint blockade therapies or multiple modalities with radiation therapy is being used. Combining the immunomodulatory effects of immune checkpoint blockade and radiation therapy provides an exciting opportunity to synergistically amplify a beneficial antitumor response [89-92]. Pretreatment with antiCTLA-4 before radiation therapy produces improved tumor controls in preclinical models, a finding supported by clinical anecdotes and early clinical evidence [93-96]. However, while combined therapy improves the clinical response rate, it also increases the toxicity of immune checkpoint blockade therapy. For example, the rate of fatigue and hypophysitis, defined as anterior pituitary hormone deficiency with side effects like headache, fatigue, general discomfort, or visual disturbances, are dramatically increased with combination therapy. The mechanism underlying hypophysitis is not clear. In a preclinical mouse model, repeated administration of antii-CTLA-4 antibodies induce hypophysitis characterized by focal lymphocytic infiltrate and antibodies in the anterior pituitary mainly against prolactin and ACTH secreting cell [97]. While combined treatment may increase antitumor efficacy, there is a gap regarding how increased systemic inflammation may further exacerbate neuroinflammation and constitutional symptoms (Figure 3). 


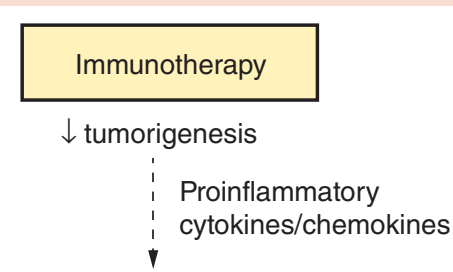

$\uparrow$ CNS inflammation

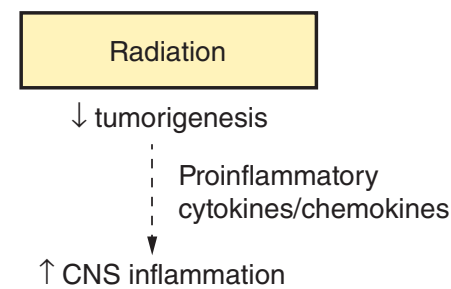

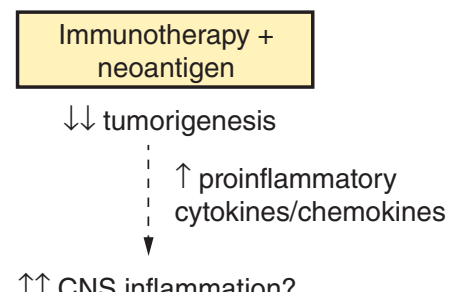

$\uparrow \uparrow$ CNS inflammation?

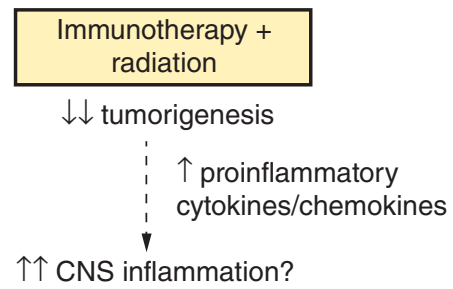

Figure 3. New therapies leading to enhanced immune activation, while beneficial for reducing tumorigenesis, may exacerbate behavioral and cognitive symptoms of cancer and cancer treatment.

\section{Conclusion}

Given the significance of cancer-related behavioral and cognitive impairments to cancer patients, it is critical to understand how novel treatment modalities may potentiate these negative effects. Little is known about the systemic and central immune-related and associated behavioral and cognitive effects of combining immune checkpoint blockade and radiation therapy. However, given the overall more robust immune response generated and the increased toxicity profile of combined treatment, it is expected that the combined modality will be associated with behavioral alterations and cognitive injury. The contribution of checkpoint inhibitor immunotherapy to the detrimental cognitive and behavioral effects of cancer and cancer treatment raises the importance of several translational foci. The preclinical and clinical data highlight the need to develop therapeutic strategies to mitigate the CNS effects of the novel immune-related treatment beneficial for tumorigenesis but not necessarily for brain function. Insight into the effects of these treatments in the context of healthy, tumor-bearing and cured individuals is especially important as patients responding to these treatments have the potential to achieve durable cancer remission requiring chronic treatment. As a result, quality-of-life issues in these survivors, while in many aspects a good problem to have, become an increasingly important clinical challenge. Based on the individual treatment responses following checkpoint inhibitor therapy seen, tailoring the type, dose and daily time for treatment based on the genetic makeup and predicted treatment response of individual patients will be critical. Besides technological advances, increased efforts are warranted as to how to best implement these new paradigms of personalized medicine in the clinic.

Checkpoint inhibitor immunotherapy allows for unique insight into the dynamics of peripheral and central immune activation in contributing to cancer-related cognitive and behavioral impairment. As we gain a better understanding of the factors underlying these impairments and how genetic substrate modulates effects, we can better approach prevention and treatment of cancer-related cognitive and behavioral impairment.

\section{Future perspective}

Checkpoint inhibitor immunotherapy, especially in combination therapy or in a multimodality regimen, represents a significant advance in tumor-directed therapy and will likely change the face of cancer survivorship in the coming years. However, current therapies only benefit a certain segment of patients and as yet, we are unable to predict which ones. Similarly, we are unable to predict which patients may be particularly vulnerable to developing CNS side effects to checkpoint inhibitor immunotherapy. Current research demonstrates the potential for these therapies to exert a central effect with significant cognitive and behavioral side effects. We believe that as these effects are better described, we will also begin to better understand the mechanisms underlying individual susceptibility. As our understanding of biomarkers to predict the success and toxicity of checkpoint inhibitor immunotherapy improves, we will begin to practice more sophisticated personalized medicine. We will be able to predict not only which patients will uniquely benefit from a given therapy, but we will also be able to predict which patients are uniquely vulnerable to side effects and may have different cost to benefit ratio of pursuing a given treatment. 
In addition to better predicting which patients may undergo significant treatment-related harm, we believe current insights will lead to effective therapy for treatment and prevention of cancer-related behavioral and cognitive impairments. Already through the understanding of the role of immune activation in mediating these effects, early studies of therapies like minocycline targeted ones to prevent or decrease central immune activation show promising results [98]. Additional clinical trials are needed to determine if adding similar interventions to new and existing therapeutic approaches can affect clinical outcomes. Achieving the best balance of immune activation for beneficial antitumor activity versus the detrimental effects to the brain is an important frontier in cancer research, with the potential to greatly improve patient quality of life.

Financial \& competing interests disclosure

GJ McGinnis is supported by an HHMI Medical Research Fellowship, the Collins Medical Trust, an RSNA Research Medical Student Grant (RMS1416) and the development account of J Raber. The tumor models and radiation treatment in authors' preclinical tumor models were supported by NCI R01 CA182311 and an instrument grant from the MJ Murdock Charitable Trust and the Providence Research Foundation. For radiation studies, J Raber is supported by NASA NNJ12ZSA001N and NNX15AK13G. The authors have no other relevant affiliations or financial involvement with any organization or entity with a financial interest in or financial conflict with the subject matter or materials discussed in the manuscript apart from those disclosed.

No writing assistance was utilized in the production of this manuscript.

\section{Executive summary}

- Fatigue, depression, anxiety, difficulty concentrating and memory impairments are commonly seen with cancer and cancer treatment.

- Peripheral mediation of central immune activation likely plays a significant role in modulating behavioral alterations and cognitive impairments seen in patients with cancer and following cancer treatment.

Immune checkpoint inhibitors \& the CNS

- Immune checkpoint inhibitors function by activating the peripheral immune system through regulation of the T-cell response.

- Human clinical trials provide accumulating evidence that systemically delivered checkpoint inhibitor immunotherapy has action against CNS metastases.

- These effects might involve direct and indirect effects of checkpoint inhibitors on immune mediators in the brain.

- CNS side effects of checkpoint inhibitor immunotherapy include fatigue, cognitive dysfunction and memory loss.

- However, there is a gap with regard to systematic studies of behavioral and cognitive outcomes.

The role of genetic factors in modulating the treatment response \& CNS side effects

- Biomarkers to tailor and predict response to treatment as well as prediction of adverse events is becoming more important in the utilization of immunotherapy.

- Known genetic risk factors like ApoE isoforms can modulate the immune system as well as vulnerability to specific adverse events, like cognitive impairment following neuroinflammation.

- ApoE4 is a risk factor for cancer- and cancer-treatment related behavioral alterations and cognitive impairments. Preclinical models to study CNS effects of immune checkpoint inhibitors \& combined immune checkpoint inhibitors

\& peripheral irradiation

- Combined immunotherapy and multimodality approaches improve tumor-related outcomes.

- Peripheral, nonbrain radiation treatment, can provoke a systemic and/or central immune response which contributes to behavioral and cognitive impairments.

- Animal models involving tumor-bearing animals as well as those that were never bearing tumors or were bearing tumors but do not bear them anymore are not required to assess effects of therapeutic interventions on the brain and evaluate the differential responses of these groups to therapy.

Conclusion

- Combined immunotherapy and multimodality approaches improve tumor-related outcomes, but might increase neuroinflammation and subsequent behavioral alterations and cognitive impairments.

- Increased efforts are warranted to enhance our understanding of behavioral and cognitive outcomes following combined immunotherapy and multimodality cancer treatment approaches and the mechanisms underlying these effects that can be used to develop therapeutic strategies to minimize or even prevent detrimental CNS effects of these novel treatments.

Future perspective

- In the future, biomarkers to predict both treatment outcome and toxicity of checkpoint inhibitor immunotherapy will allow for best utilization of this novel treatment approach. 
Open access

This work is licensed under the Attribution-NonCommercial-NoDerivatives 4.0 Unported License. To view a copy of this license, visit http://creativecommons.org/licenses/by-nc-nd/4.0/

\section{References}

Papers of special note have been highlighted as: $\bullet$ of interest; $\bullet \bullet$ of considerable interest

1 Ahles TA, Root JC, Ryan EL. Cancer- and cancer treatment-associated cognitive change: an update on the state of the science. J. Clin. Oncol. 30, 3675-3686 (2012).

2 Jim HSL, Donovan KA, Small BJ, Andrykowski MA, Munster PN, Jacobsen PB. Cognitive functioning in breast cancer survivors: a controlled comparison. Cancer 115, 1776-1783 (2009).

3 Bower JE, Ganz PA, Irwin MR, Kwan L, Breen EC, Cole SW. Inflammation and behavioral symptoms after breast cancer treatment: do fatigue, depression and sleep disturbance share a common underlying mechanism? J. Clin. Oncol. 29, 3517-3522 (2011).

4 Bower JE, Ganz PA, Tao ML et al. Inflammatory biomarkers and fatigue during radiation therapy for breast and prostate cancer. Clin. Cancer Res. 15(17), 5534-5540 (2009).

5 Von Ah D, Habermann B, Carpenter JS, Schneider BL. Impact of perceived cognitive impairment in breast cancer survivors. Eur. J. Oncol. Nurs. 17(2), 236-241 (2013).

6 Ganz PA, Kwan L, Castellon SA et al. Cognitive complaints after breast cancer treatments: examining the relationship with neuropsychological test performance. J. Natl Cancer Inst. 105(11), 791-801 (2013).

7 Pyter LM, El Mouatassim Bih S, Sattar H, Prendergast BJ. Peripheral tumors alter neuroinflammatory responses to lipopolysaccharide in female rats. Brain Res. 1552, 55-63 (2014).

8 Haroon E, Raison CL, Miller AH. Psychoneuroimmunology meets neuropsychopharmacology: translational implications of the impact of inflammation on behavior. Neuropsychopharmacol. 37(1), 137-162 (2012).

9 Norden DM, Mccarthy DO, Bicer S et al. Ibuprofen ameliorates fatigue- and depressive-like behavior in tumor-bearing mice. Life Sci. $143,65-70$ (2015).

10 Capuron L, Miller AH. Immune system to brain signaling: neuropsychopharmacological implications. Pharmacol. Ther. 130(2), 226-238 (2011).

11 Ramesh G, MacLean AG, Philipp MT. Cytokines and chemokines at the crossroads of neuroinflammation, neurodegeneration, and neuropathic pain. Mediators of Inflammation 130, 480739 (2013).

12 Zenaro E, Pietronigro E, Bianca VD et al. Neutrophils promote Alzheimer's disease-like pathology and cognitive decline via LFA-1 integrin. Nat. Med. doi:10.1038/nm.3913 (2015) (Epub ahead of print).

13 Torres-Platas SG, Cruceanu C, Chen GG, Turecki G, Mechawar N. Evidence for increased microglial priming and macrophage recruitment in the dorsal anterior cingulate white matter of depressed suicides. Brain Behav. Immun. 42, 50-59 (2014).

14 Pertl MM, Hevey D, Boyle NT et al. C-reactive protein predicts fatigue independently of depression in breast cancer patients prior to chemotherapy. Brain Behav. Immun. 34, 108-119 (2013).

15 Renner M, Feng R, Springer D et al. A murine model of peripheral irradiation-induced fatigue. Behav. Brain Res. 307, 218-226 (2016)

16 Saligan LN, Kim HS. A systematic review of the association between immunogenomic markers and cancer-related fatigue. Brain Behav. Immun. 26(6), 830-848 (2012).

17 Wood LJ, Weymann K. Inflammation and neural signaling: etiologic mechanisms of the cancer treatment-related symptom cluster. Curr. Opin. Support. Palliat. Care 7(1), 54-59 (2013).

18 Miller AC, Raison C. The role of inflammation in depression: from evolutionary imperative to modern treatment target. Nat. Rev. Immunol. 16, 22-34 (2015).

19 Raison C, Miller A. The evolutionary significance of depression in Pathogen Host Defense (PATHOS-D). Mol. Psychiatry 18, 15-37 (2013).

20 Dantzer R. Cytokine-induced sickness behaviour: a neuroimmune response to activation of innate immunity. Eur. J. Pharamacol. 500, 399-411 (2004).

21 Denardo DG, Brennan DJ, Rexhepaj E et al. Leukocyte complexity predicts breast cancer survival and functionally regulates response to chemotherapy. Cancer Discov. 1(1), 54-67 (2011).

22 Galon J, Costes A, Sanchez-Cabo F et al. Type, density and location of immune cells within human colorectal tumors predict clinical outcome. Science (New York) 313, 1960-1964 (2006).

23 Huang RR, Jalil J, Economou JS et al. CTLA-4 blockade induces frequent tumor infiltration by activated lymphocytes regardless of clinical responses in humans. Clin. Cancer Res. 17(12), 4101-4109 (2011).

24 Hui E, Cheung J, Zhu J et al. T-cell costimulatory receptor CD28 is a primary target for PD-1-mediated inhibition. Science 4, eaaf1292 (2017). 
25 Kamphorst AO, Kamphorst AO, Wieland A et al. Rescue of exhausted CD8 T cells by PD-1 - targeted therapies is CD28-dependent. Science 355(6332), 1423-1427 (2017).

26 Topalian SL, Hodi FS, Brahmer JR et al. Safety, activity and immune correlates of anti-PD-1 antibody in cancer. N. Engl. J. Med. 366, 2443-2454 (2012).

27 Fecci P, Ochiai H, Mitchell D et al. Systemic CTLA-4 blockade ameliorates glioma-induced changes to the CD4+ T cell compartment without affecting regulatory T-cell function. Clin. Cancer Res. 13, 2158-2167 (2007).

28 Zeng J, See A, Phallen J et al. Anti-PD-1 blockade and stereotactic radiation produce long-term survival in mice with intracranial gliomas. Int. J. Radiat. Oncol. Biol. Phys. 86, 343-349 (2013).

29 Reardon D, Gokhale P, Klein S, Ligon K, Rodig S, Ramkissoon S. Glioblastoma eradication following immune checkpoint blockade in an orthotopic, immunocompetent model. Cancer Immunol. Res. 4, 124-135 (2016).

30 Goldberg SB, Gettinger SN, Mahajan A et al. Pembrolizumab for patients with melanoma or non-small-cell lung cancer and untreated brain metastases: early analysis of a nonrandomized, open-label, Phase II trial. Lancet Oncol. 17(7), 976-983 (2016).

31 Rizvi NA, Mazières J, Planchard D et al. Activity and safety of nivolumab, an anti-PD-1 immune checkpoint inhibitor, for patients with advanced, refractory squamous non-small-cell lung cancer (CheckMate 063): a Phase II, single-arm trial. Lancet Oncol. 16(3), 257-265 (2015).

32 Dudnik E, Yust-Katz S, Nechushtan H et al. Intracranial response to nivolumab in NSCLC patients with untreated or progressing CNS metastases. Lung Cancer 98, 114-117 (2016).

33 Baruch K, Deczkowska A, Rosenzweig N et al. PD-1 immune checkpoint blockade reduces pathology and improves memory in mouse models of Alzheimer's disease. Nat. Med. 22, 135-137 (2016).

34 Tarhini A. Immune-mediated adverse events associated with ipilimumab ctla- 4 blockade therapy: the underlying mechanisms and clinical management. Scientifica (Cairo) 2013, 857519 (2013).

35 Blansfield JA, Beck KE, Tran K et al. Cytotoxic T-lymphocyte-associated Antigen-4 blockage can induce autoimmune hypophysitis in patients with metastatic melanoma and renal cancer. J. Immunother. 28, 593-598 (2005).

36 Romano E, Kusio-Kobialka M, Foukas P et al. Iplimumab-dependent cell-mediated cytotoxicity of regulatory T cells ex vivo by nonclassical monocytes in melanoma patients. Proc. Natl Acad. Sci. USA 112, 6140-6145 (2015).

37 Sunshine J, Taube J. PD-1/PD-L1 inhibitors. Curr. Opin. Pharmacol. 23, 32-38 (2015).

38 Postow MA, Chesney J, Pavlick AC et al. Nivolumab and ipilimumab versus ipilimumab in untreated melanoma. N. Engl. J. Med. 372(21), 2006-2017 (2015).

39 Larkin J, Chiarion-Sileni V, Gonzalez R et al. Combined nivolumab and ipilimumab or monotherapy in untreated melanoma. N. Engl. J. Med. 373, 23-34 (2015).

40 Fabrizio D. A fuller picture. The Scientist 4, 29-30 (2017).

41 Azvolinsky A. Resist or desist. The Scientist 4, 41-47 (2017).

42 Madhusoodanian J. Cures by the clock. The Scientist 4, 55-59 (2017).

43 Berkowitz R. Special delivery. The Scientist 4, 66-69 (2017).

44 Offord C. Safety belts. The Scientist 4, 73-75 (2017).

45 Farrer LA, Cupples LA, Haines JL et al. Effects of age, sex and ethnicity on the association between apolipoprotein E genotype and Alzheimer's disease. A meta-analysis. J. Am. Med. Assoc. 278, 1349-1356 (1997).

46 Raber J. Differential gene actions of polymorphic alleles at the APOE locus; potential role of androgens and androgen receptor-mediated signaling. Science of Aging (SAGE) Knowledge Environment (KE) 2004(11), 2 (2004).

47 Raber J, Huang Y, Ashford J. ApoE genotype accounts for the vast majority of AD risk and AD pathology. Neurobiol. Aging 25, 641-650 (2004).

48 Brichtova E, Kozak L. Apolipoprotein E genotype and traumatic brain injury in children-association with neurological outcome. Childs Nerv. Syst. 24, 349-356 (2008).

49 Jordan BD, Relkin NR, Ravdin LD, Jacobs AR, Bennett A, Gandy S. Apolipoprotein E e4 associated with chronic traumatic brain injury in boxing. J. Am. Med. Assoc. 278, 136-140 (1997).

50 Hui DY, Harmony JaK, Innerarity TL, Mahley RW. Immunoregulatory plasma lipoproteins: role of apoprotein E and apoprotein B. J. Biol. Chem. 255(24), 11775-11781 (1980).

51 Mcgeer PL, Walker DG, Pitas RE, Mahley RW, Mcgeer EG. Apolipoprotein E4 (ApoE4) but not ApoE3 or ApoE2 potentiates beta-amyloid protein activation of complement in vitro. Brain Res. 749, 135-138 (1997).

52 Corder EH, Robertson K, Lannfelt L et al. HIV-infected subjects with the E4 allele for APOE have excess dementia and peripheral neuropathy. Nat. Med. 4(10), 1182-1184 (1998).

53 Burt T, Agan B, Marconi V et al. Apolipoprotein (apo) E4 enhances HIV-1 cell entry in vitro, and the APOE $\varepsilon 4 / \varepsilon 4$ genotype accelerates HIV disease progression. Proc. Natl Acad. Sci. USA 105, 8718-8723 (2008). 
54 Itzhaki R. Herpes simplex viris type I and Alzheimer's disease: increase evidence for a major role of the virus. Front. Aging Neurosci. 6, 202 (2014).

55 Trompet S, Jukema J, Katan M et al. Apolipoprotein E genotype, plasma cholesterol and cancer: a Mendelian randomization study. Am. J. Epidemiol. 170, 1415-1421 (2009).

56 Grant W. A multicountry ecological study of risk-modifying factors for prostate cancer: apolipoprotein E epsilon 4 as a risk factor and cereals as a risk reduction factor. Anticancer Res. 30, 189-199 (2010).

57 De Feo E, Simone B, Persiani R et al. A case-control study on the effect of Apolipoprotein E genotypes on gastric cancer risk and progression. BMC Cancer 12, 494 (2012).

58 Ifere G, Desmond R, Demark-Wahnefried W, Nagy T. Apolipoprotein E gene polymorphism influences aggressive behavior in prostate cancer cells by deregulating cholesterol homeostasis. Int. J. Oncol. 43(4), 1002-1010 (2013).

59 Chen Y-C, Pohl G, Wang T-L et al. Apolipoprotein E is required for cell proliferation and survival in ovarian cancer. Cancer Res. 65, 331 (2005).

60 Mcginnis G, Yu B, Ransom C et al. E4 cancer survivors show better fall and functional status outcomes after receiving exercise interventions than non-E4 cancer survivors. Presented at: American Society for Radiation Oncology (ASTRO) Annual Meeting. San Diego, CA, USA, 24-27 September 2017.

61 Juhasz G, Gonda X, Hullam G et al. Variability in the effect of 5-HTTLPR on depression in a large European population: the role of age, symptom profile, type and intensity of life stressors. PLoS ONE 10, e0116316 (2015).

62 Udina M, Moreno-Espana J, Navines R et al. Serotonin and interleukin-6: the role of geentic polymorphisms in IFN-induced neuropsychiatrci symptoms. Psychoneuroendocrinol. 38, 1803-1813 (2013).

63 Sarginson J, Deakin JF, Anderson I et al. Neuronal nitric oxide aynthase (NOS1) polymoprhisms interact with financial hardship to affect depression risk. Neuropsychopharmaco. 39, 2857-2886 (2014)

64 Smith K, Norris S, O'Farrrelly C, O'mara S. Risk factors for the development of depression in patients with hepatitits C taking interferon-alpha. Neuropsychiatr. Dis. Treat. 7, 275-292 (2011).

65 Raber J, Rola R, Lefevour A et al. Radiation-induced cognitive impairments are associated with changes in indicators of hippocampal neurogenesis. Radiat. Res. 162, 39-47 (2004).

66 Winocur G, Henkelman M, Woltowicz J, Zhang H, Binns M, Tannock I. The effects of chemotherapy on cognitive function in a mouse model: a prospective study. Clin. Cancer Res. 18, 3112-3132 (2012).

67 Shi Q, Chowdhury S, Ma R et al. Complement C3 deficiency protects against neurodegeneration in aged plaque-enriched APP/PS1 mice. Sci. Trans. Med. 31, 9 (2017).

68 Wolf S, Boddeke H, Kettenmann H. Microglia in physiology and diease. Ann. Rev. Physiol. 79, 619-643 (2017).

69 Abayomi OK. Pathogenesis of irradiation-induced cognitive dysfunction. Acta Oncol. 35(6), 659-663 (1996).

70 Mcginnis GJ, Friedman D, Young KH et al. Neuroinflammatory and cognitive consequences of combined radiation and immunotherapy in a novel preclinical model. Oncotarget 8(6), 9155-9173 (2017).

•• Described an animal tumor model of behavioral and cognitive response to tumor-directed interventions.

71 Moravan M, Olschowka J, Williams J, O’ Banion M. Cranial irradiation leads to acute and persistent neuroinflammation with delayed increases in T-cell infiltration and CD11c expression in C57BL/6 mouse brain. Radiat. Res. 176, 459-473 (2011).

72 Qin L, Wu X, Block M et al. Systemic LPS causes chronic neuroinflammation and progressive neurodegeneration. Glia 55, 453-462 (2007).

73 Rola R, Fishman K, Baure J et al. Hippocampal neurogenesis and neuroinflammation after cranial irradiation with 56Fe particles. Radiat. Res. 169, 626-632 (2008).

74 Rosi S, Ramirez-Amaya V, Vazdarjanova A et al. Accuracy of hippocampal network activity is disrupted by neuroinflammation: rescue by memantine. Brain 1, 1-14 (2009).

75 Monje ML, Toda H, Palmer TD. Inflammatory blockade restores adult hippocampal neurogenesis. Science (New York) 302, 1760-1765 (2003).

76 Akiyama H, Barher S, Barnum S et al. Inflammation and Alzheimer's disease. Neuroinflammation working group. Neurobiol. Aging 21(3), 383-421 (2000).

77 Huenhnchen P, Prozorovski T, Klaissle P et al. Modulation of adult hippocampal neurogenesis during myelin-directed autoimmne neuroinflammation. Glia 59, 132-142 (2011).

78 Acevedo SF, Mcginnis GJ, Raber J. Effects of 137Cs gamma irradiation on cognitive performance and measures of anxiety in Apoe-/and wild-type female mice. Radiat. Res. 428, 422-428 (2008).

79 Raber J, Rola R, Lefevour A et al. Radiation-induced cognitive impairments are associated with changes in indicators of hippocampal neurogenesis. Radiat. Res. 162, 39-47 (2004).

80 Raber J, Villasana L, Rosenberg J, Zou Y, Huang TT, Fike JR. Irradiation enhances hippocampus-dependent cognition in mice deficient in extracellular superoxide dismutase. Hippocampus 21(1), 72-80 (2011). 
81 Monje ML, Toda H, Palmer TD. Inflammatory blockade restores adult hippocampal neurogenesis. Science 302(5651), 1760-1765 (2003).

82 Morganti JM, Jopson TD, Liu S, Gupta N, Rosi S. Cranial irradiation alters the brain's microenvironment and permits CCR2+ macrophage infiltration. PLoS ONE 9(4), e93650 (2014).

83 Lee W, Sonntag W. Irradiation induces regionally specific alterations in proinflammatory environments in rat brain. Int. J. Radiat. Biol. 86, 132-144 (2010).

84 Lee $\mathrm{Y}$, Auh SL, Wang Y et al. Therapeutic effects of ablative radiation on local tumor require CD8+ T cells: changing strategies for cancer treatment. 114, 589-595 (2009).

85 Stamell EF, Wolchok JD, Gnjatic S, Lee NY, Brownell I. The abscopal effect associated with a systemic antimelanoma immune response. Int. J. Radiat. Oncol. Biol. Phys. 85(2), 293-295 (2013).

86 Kanagavelu S, Gupta S, Wu X et al. In vivo effects of lattice radiation therapy on local and distant lung cancer: potential role of immunomodulation. Radiat. Res. 182(2), 149-162 (2014).

87 Roses RE, Datta J, Czerniecki BJ. Radiation as immunomodulator: implications for dendritic cell-based immunotherapy. Radiat. Res. 182(2), 211-218 (2014).

88 Curran MA, Montalvo W, Yagita H, Allison JP. PD-1 and CTLA-4 combination blockade expands infiltrating T cells and reduces regulatory T and myeloid cells within B16 melanoma tumors. Proc. Natl Acad. Sci. USA 107(9), 4275-4280 (2010).

89 Gough M, Crittenden M, Sarff M et al. Adjuvant therapy with agonistic antibodies to CD134 (OX40) increases local control following surgical or radiation therapy of cancer in mice. J. Immunother. 33, 798-809 (2010).

90 Demaria S, Kawashima N, Yang AM et al. Immune-mediated inhibition of metastases after treatment with local radiation and CTLA-4 blockade in a mouse model of breast cancer. Clin. Cancer Res. 11(2 Pt 1), 728-734 (2005).

91 Deng L, Liang H, Burnette B et al. Irradiation and anti-PD-L1 treatment synergistically promote antitumor immunity in mice. J. Clin. Invest. 124, 687-695 (2014).

92 Sun B, Chen L, Wu Q, Wang H. Suppression of inflammatory response by flurbiprofen following focal cerebral ischemia involves the NF-kB signaling pathway. Int. J. Clin. Exp. Med. 7(9), 3087-3095 (2014).

93 Young KH, Baird JR, Savage T et al. Optimizing timing of immunotherapy improves control of tumors by hypofractionated radiation therapy. PLoS ONE 11(6), e0157164 (2016).

94 Postow MA, Callahan MK, Barker CA et al. Immunologic correlates of the abscopal effect in a patient with melanoma. N. Engl. J. Med. 366, 925-931 (2012).

95 Hiniker SM, Chen DS, Reddy S et al. A systemic complete response of metastatic melanoma to local radiation and immunotherapy. Transl. Oncol. 5, 404-407 (2012).

96 Barker CA, Postow MA, Khan SA et al. Concurrent radiotherapy and ipilimumab immunotherapy for patients with melanoma. Cancer Immunol. Res. 1(2), 92-98 (2013).

97 Iawama S, De Remigis A, Callahan M, Slovin S, Wolchok J, Caturegli P. Pituitary expression of CTLA-4 mediates hypophysitis secondary to administration of CTLA-4 blocking antibody. Sci. Trans. Med. 6, 230ra245 (2014).

98 Vichaya EG, Vermeer DW, Christian DL et al. Neuroimmune mechanisms of behavioral alterations in a syngeneic murine model of human papilloma virus-related head and neck cancer. Psychoneuroendocrinol. 79, 59-66 (2017).

99 Hodi F, O'day S, Mcdermott D et al. Improved survival with ipilimumab in patients with metastatic melanoma. N. Engl. J. Med. 363 , 711-723 (2010).

100 Garon E, Rizvi N, Hui R et al. Pembrolizumab for the treatment of non-small-cell lung cancer. N. Engl. J. Med. 372, 2018-2028 (2015).

101 Weber J, D'angelo S, Minor D et al. Nivolumab versus chemotherapy in patients with advanced melanoma who progressed after anti-CTLA-4 treatment (CheckMate 037): a randomized, controlled, open-label, Phase III trial. Lancet Oncol. 16, 375-384 (2015).

102 Vardy JL, Dhillon HM, Pond GR et al. Cognitive function in patients with colorectal cancer who do and do not receive chemotherapy: a prospective, longitudinal, controlled study. J. Clin. Oncol. 33, 4085-4092 (2015).

\section{-. Describes ApoE4-associated cognitive impairments in cancer and cancer treatment.}

103 Amidi A, Agerbæk M, Wu LM et al. Changes in cognitive functions and cerebral grey matter and their associations with inflammatory markers, endocrine markers and APOE genotypes in testicular cancer patients undergoing treatment. Brain Imaging Behav. 11(3), 769-783 (2016).

-• Describes ApoE4-associated cognitive impairments in cancer and cancer treatment.

104 Koleck TA, Bender CM, Sereika SM et al. Apolipoprotein E genotype and cognitive function in postmenopausal women with early-stage breast cancer. Oncol. Nurs. Forum 41, E313-E325 (2014).

-• Describes ApoE4-associated cognitive impairments in cancer and cancer treatment.

105 Ahles T, Li Y, Mcdonald B et al. Longitudinal assessment of cognitive changes associated with adjuvant treatment for breast cancer: the impact of APOE and smoking. Psycho-Oncol. 23, 1382-1390 (2014).

-• Describes ApoE4-associated cognitive impairments in cancer and cancer treatment. 
106 Lengacher CA, Reich RR, Kip KE et al. Moderating effects of genetic polymorphisms on improvements in cognitive impairment in breast cancer survivors participating in a 6-week mindfulness-based stress reduction program. Biol. Res. Nurs. 17, 393-404 (2015).

-• Describes ApoE4-associated cognitive impairments in cancer and cancer treatment.

107 Ahles TA, Saykin AJ, Noll WW et al. The relationship of APOE genotype to neuropsychological performance in long-term cancer survivors treated with standard dose chemotherapy. Psychooncol. 12, 612-619 (2003).

108 Fang CK, Chen HW, Chiang IT et al. Mirtazapine inhibits tumor growth via immune response and serotonergic system. PLoS ONE 7 , e38886 (2012).

- Described an animal tumor model of behavioral and cognitive response to tumor-directed interventions.

109 Yang M, Kim J-S, Kim J et al. Acute treatment with methotrexate induces hippocampal dysfunction in a mouse model of breast cancer. Brain Res. Bull. 89, 50-56 (2012).

- Described an animal tumor model of behavioral and cognitive response to tumor-directed interventions. 